\title{
Multidisciplinary management of soft tissue sarcoma
}

Soft tissue sarcoma (STS) is a rare disease that represents $1 \%$ of all solid tumors in adults and encompasses over 50-70 different histologic subtypes, each with distinct genetic aberrations, clinical behavior and response to treatment. To add to their complexity, tumors can occur in different body locations including the extremity, trunk and retroperitoneum. In general, STS can be aggressive and are potentially deadly. Due to its rarity, the vast majority of clinicians know very little about STS and as a result, misdiagnoses can be made and inappropriate treatment may be given, all to the patient's detriment.

This issue of Chinese Clinical Oncology (CCO) focuses on STS and strives to educate and update readers on the current multidisciplinary management of this complex disease. We begin with a broad overview of STS imaging and pathology, followed by current staging and "tools" such as nomograms that are available to help the clinician to predict outcome. The mainstay of treatment for the majority of localized STS is surgery and in this issue, surgical management is addressed separately by body location: extremity, trunk, and retroperitoneum. One article specifically discusses surgical palliation in retroperitoneal sarcoma. For high risk and advanced/metastatic STS, we cover the general principles of radiation and systemic therapy. There is also a separate article on the "next frontiers" in systemic therapy (e.g., targeted therapies) and one article focused just on NY-ESO-1, a recently recognized immune target that has strong therapeutic potential for select STS subtypes. For all subtypes, post-treatment surveillance is important and recommendations for this are summarized. Finally, we conclude with an exploration of the current status and future directions of STS management in Asia. In all of the articles in this issue of $C C O$, the importance of multidisciplinary care is emphasized.

We would like to thank $C C O$ for the opportunity to put together this issue on STS. We invited specialists from all the clinical disciplines that are involved in the day-to-day care of STS patients to contribute articles. In total, the authors represent 12 different institutions around the world, including 5 from the United States of America, 4 from Asia, 2 from Europe and 1 from Canada. We are very proud of the multidisciplinary, multi-institutional collaboration inherent in this issue. For such a rare and complex disease, we believe that this type of global collaboration is critical to move forward in the field with our understanding of STS and ultimately, the development of better treatment.

\section{Acknowledgements}

None.

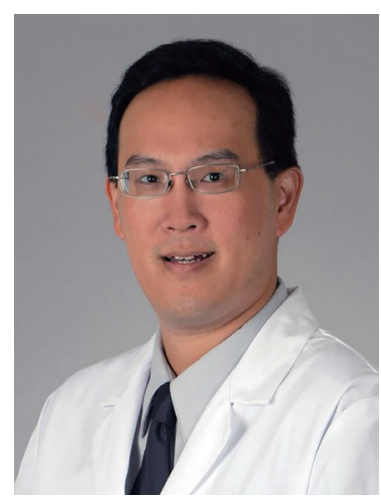

William W. Tseng

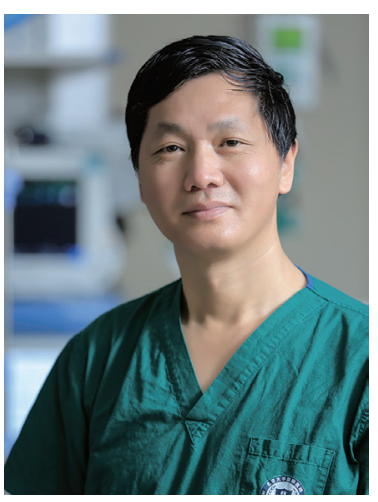

Cheng-Hua Luo

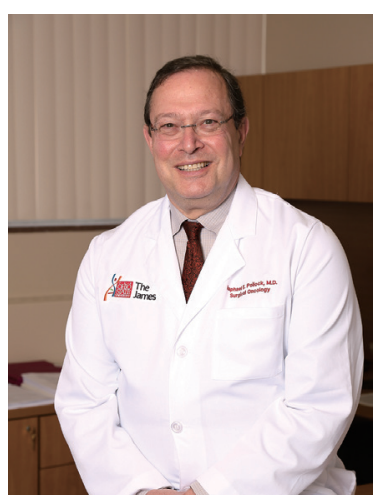

Raphael E. Pollock 
William W. Tseng, MD

University of Southern California, Los Angeles, CA, USA. (Email: William.Tseng@med.usc.edu) Cheng-Hua Luo, MD

Peking University International Hospital, Beijing 102206, China. (Email: LUOCHENGHUA@pkuih.edu.cn)

Raphael E. Pollock, MD, PhD

Obio State University, Columbus, OH, USA. (Email: Raphael.Pollock@osumc.edu)

doi: 10.21037/cco.2018.08.09

Conflicts of Interest: The authors have no conflicts of interest to declare.

View this article at: http://dx.doi.org/10.21037/cco.2018.08.09

Cite this article as: Tseng WW, Luo CH, Pollock RE. Multidisciplinary management of soft tissue sarcoma. Chin Clin Oncol 2018;7(4):34. doi: 10.21037/cco.2018.08.09 